\title{
BMJ Open Risk of out-of-hospital cardiac arrest among sepsis survivors in Taiwan: analysis of a nationwide population- based cohort
}

\author{
Wan-Ting Hsu (D) , ${ }^{1}$ Charles Fox Sherrod, ${ }^{2}$ Babak Tehrani, ${ }^{2}$ Alexa Papaila, ${ }^{2}$ \\ Lorenzo Porta, ${ }^{3}$ Tzu-Chun Hsu, ${ }^{4}$ Wang-Huei Sheng, ${ }^{5}$ Chien-Chang Lee ${ }^{4,6,7}$
}

To cite: Hsu W-T, Sherrod CF, Tehrani B, et al. Risk of outof-hospital cardiac arrest among sepsis survivors in Taiwan: analysis of a nationwide populationbased cohort. BMJ Open 2021;11:e051502. doi:10.1136/ bmjopen-2021-051502

- Prepublication history and additional supplemental material for this paper are available online. To view these files, please visit the journal online. (http://dx.doi.org/10.1136/ bmjopen-2021-051502).

Received 25 March 2021 Accepted 27 July 2021

Check for updates

(C) Author(s) (or their employer(s)) 2021. Re-use permitted under CC BY-NC. No commercial re-use. See rights and permissions. Published by BMJ.

For numbered affiliations see end of article.

\section{Correspondence to} Professor Chien-Chang Lee, Emergency Medicine, National Taiwan University Hospital,

Taipei, Taiwan;

cclee100@gmail.com

\section{ABSTRACT}

Objectives There is minimal literature examining the association of sepsis with out-of-hospital cardiac arrest (OHCA). Using a large national database, we aimed to quantify the risk of OHCA among sepsis patients after hospital discharge.

Design Population-based cohort study.

Setting Nationwide sepsis cohort retrieved from the National Health Insurance Research Database of Taiwan between 2000 and 2013.

Participants We included 17304 patients with sepsis. After hospital discharge, 144 patients developed OHCA within 30 days and 640 between days 31 and 365 .

Primary and secondary outcome measures The main outcomes were OHCA events following hospital discharge for sepsis. To evaluate the independent association between sepsis and OHCA after a sepsis hospitalisation, we constructed two non-sepsis comparison cohorts using risk set sampling and propensity score matching techniques (non-infection cohort, non-sepsis infection cohort). We plotted the daily number and daily risk of OHCA within 1 year of hospital discharge between sepsis and matched non-sepsis cohorts. We used Cox regression to evaluate the risk of early and late OHCA, comparing sepsis to non-sepsis patients.

Results Compared with non-infected patients, sepsis patients had a higher rate of early (HR 1.66, 95\% Cl: 1.27 to 2.16 ) and late (HR $1.19,95 \% \mathrm{Cl}: 1.06$ to 1.33 ) OHCA events. This association was independent of age, sex or cardiovascular history. Compared with non-sepsis patients with infections, sepsis patients had a higher rate of both early (HR 1.28, 95\% Cl: 1.00 to 1.63) and late (HR 1.13, $95 \% \mathrm{Cl}: 1.01$ to 1.27$)$ OHCA events, especially among patients with cardiovascular disease (OR 1.35, 95\% Cl: 1.01 to 1.81).

Conclusions Sepsis patients had increased risk of OHCA compared with matched non-sepsis controls, which lasted up to 1 year after hospital discharge.

\section{INTRODUCTION}

Cardiac arrest is a serious complication of sepsis and septic shock. In a 2019 national database in the USA with 8058091 patients, $2.3 \%$ of patients treated for sepsis suffered from in-hospital cardiac arrest (IHCA). ${ }^{1}$ Data
Strengths and limitations of this study

- A major strength is the use of a large populationbased sepsis cohort.

- This study was performed using a large, nationally representative longitudinal sampling database to identify cases and extract information about relatively rare events with sufficiently powered and matched controls.

- This study is observational in nature and is subject to confounding.

- Two non-sepsis comparison cohorts using risk set sampling and propensity score matching techniques were constructed to ameliorate confounding and bias.

have shown that patients with sepsis who experience IHCA are less likely to obtain return of spontaneous circulation, particularly among patients with pneumonia-related sepsis who more frequently present with unshockable rhythms. $^{23}$

Whereas only $13 \%$ of patients survive IHCAs 1 year after the event, far fewer (roughly $7 \%$ ) survive out-of-hospital cardiac arrest (OHCA).${ }^{4}$ No large national studies investigating the occurrence of in-hospital sepsis as a risk factor in the subsequent occurrence of OHCA have been published. Among limited data that have been published regarding patients treated for in-hospital sepsis who then subsequently suffered OHCAs, most of the information were derived from studies on IHCA. ${ }^{6-8}$ Data that better characterise the relationship between sepsis and subsequent OHCA could identify populations that may benefit from preventive interventions, including increased awareness of risks (both for patients and their outpatient providers), initiation of cardioprotective pharmacotherapies, pursuit of risk stratification testing and ultimately reductions in morbidity and 
mortality from sepsis-associated cardiac arrest. ${ }^{9}{ }^{10}$ To this end, our study aimed to evaluate the risk of OHCA after being hospitalised with sepsis by comparing the incidence of OHCA in sepsis patients with the incidence of OHCA in two types of matched cohorts: hospitalised nonsepsis patients with an infection and hospitalised patients without an infection.

\section{METHODS}

\section{Design and study population}

This study analysed data from a population-based cohort in Taiwan that included all identified sepsis patients treated in emergency departments (EDs) and hospitals between 2001 and 2013. The study was conducted using the National Health Insurance Research Database (NHIRD) of Taiwan, which is a single-payer mandatory health insurance system that covers more than $99 \%$ of the 23 million people residing in Taiwan. The database used a systematic sampling strategy to identify one million participants who represented the demographic and geographic distribution of Taiwan, linked in chronological order to provide a temporal sequence of all health service utilisation. This sample was referred to as the Longitudinal Health Insurance Database. The comprehensive NHIRD is regularly maintained and cross-checked to ensure validity and accuracy of diagnoses. We reviewed subsets from the database that included electronic records for outpatient and inpatient demographics and diagnoses (note: since 2000, all diagnostic codes included in the database have used the International Classification of Diseases, Ninth Revision, Clinical Modification (ICD9-CM) system). The institutional review board of the National Taiwan University Hospital deemed this study exempt from patient consent because the NHIRD is an anonymised electronic database study.

\section{Identification of sepsis cases}

To identify patients with sepsis in our database, we used the widely validated Martin criteria to select all cases with explicit ICD-9-CM codes for sepsis in combination with ICD-9-CM codes for acute organ dysfunction. ${ }^{11}$ In accordance with the Sepsis-3 definition, sepsis was defined as a life-threatening organ dysfunction caused by a dysregulated host response to infection. ${ }^{12}$ Sepsis ICD-9-CM codes included sepsis (038 septicaemia, 020.0 septicaemic), severe sepsis (995.92), septic shock (785.52), bacteraemia (790.7) and systemic fungal infections (117.9 disseminated fungal infection, 112.5 disseminated candida infection, 112.81 disseminated fungal endocarditis). Seven acute organ dysfunctions were evaluated for this study: cardiovascular (including shock), respiratory, central nervous system, haematological, hepatic, renal and metabolic. The ICD-9-CM codes used for identification of acute organ dysfunction are listed in online supplemental appendix 1. NHIRD has been proven to be a useful source in the epidemiology of sepsis that have been widely cited and validated. ${ }^{13-16}$

\section{Covariates}

Covariate data were also collected for analysis, including demographic information, presence of pre-existing comorbidity and infection source. We defined the index date as the date of hospital discharge. As the aim of the study was to assess the risk of OHCA after discharge from a sepsis hospitalisation, only patients who survived to discharge were included for analysis. For cases with more than one sepsis event per year, recurrent sepsis admissions were not factored into our analysis. Patients were followed from the index date to whichever event occurred first: OHCA, death, termination of health insurance coverage or the end of the study period. The time frame of data collection and patient follow-up is shown in online supplemental figure 1 .

\section{Construction of the 2 non-sepsis comparison cohorts}

To ascertain the independent association between sepsis hospitalisation and incident OHCA, we constructed two non-sepsis comparison cohorts using risk set sampling in combination with propensity score (PS) matching techniques. The non-sepsis comparison cohorts were constructed using a two-stage procedure. In the first stage, we used risk set sampling to select 100 non-sepsis patients for each sepsis case that were matched according to admission date, 5-year age group, sex and Charlson Comorbidity Score quartile $(0,1-2,3-4$ and $\geq 5) .{ }^{1718}$ In the second stage, we created a PS consisting of a comprehensive set of covariates associated with sepsis. We performed 1:1 PS matching using a greedy algorithm to construct non-sepsis comparison cohorts. Component variables with relative weights of the two PS models is shown in online supplemental table 1. For comparison cohort 1 , we selected hospitalised non-sepsis medical patients that did not have a diagnostic code of infection as noninfected patients. For comparison cohort 2, we selected hospitalised non-sepsis patients with a diagnostic code of infection. Patients with a diagnostic code of infection in the primary and secondary diagnosis, but without any diagnoses of organ dysfunction were eligible for inclusion in comparison cohort 2. To evaluate the success of the matching process, we calculated the standardised difference of matching covariates (online supplemental figure 2) and compared the PS distribution before and after PS matching (online supplemental figure 3) between the sepsis cohort and the non-sepsis comparison cohort. The ICD-9-CM codes have been previously validated for identification of acute organ dysfunction (online supplemental appendix 1). ${ }^{13-16}$ The cohort's construction process is shown in figure 1 . To further investigate the differences in the rate of OHCA between patients with sepsis and septic shock, we conducted a sensitivity analysis for patients with septic shock using the Sepsis-2-definition exclusively.

\section{Outcome definition}

All emergency records associated with OHCA within 1 year of sepsis hospitalisation were identified. OHCA was identified by the primary diagnosis code for cardiac arrest 


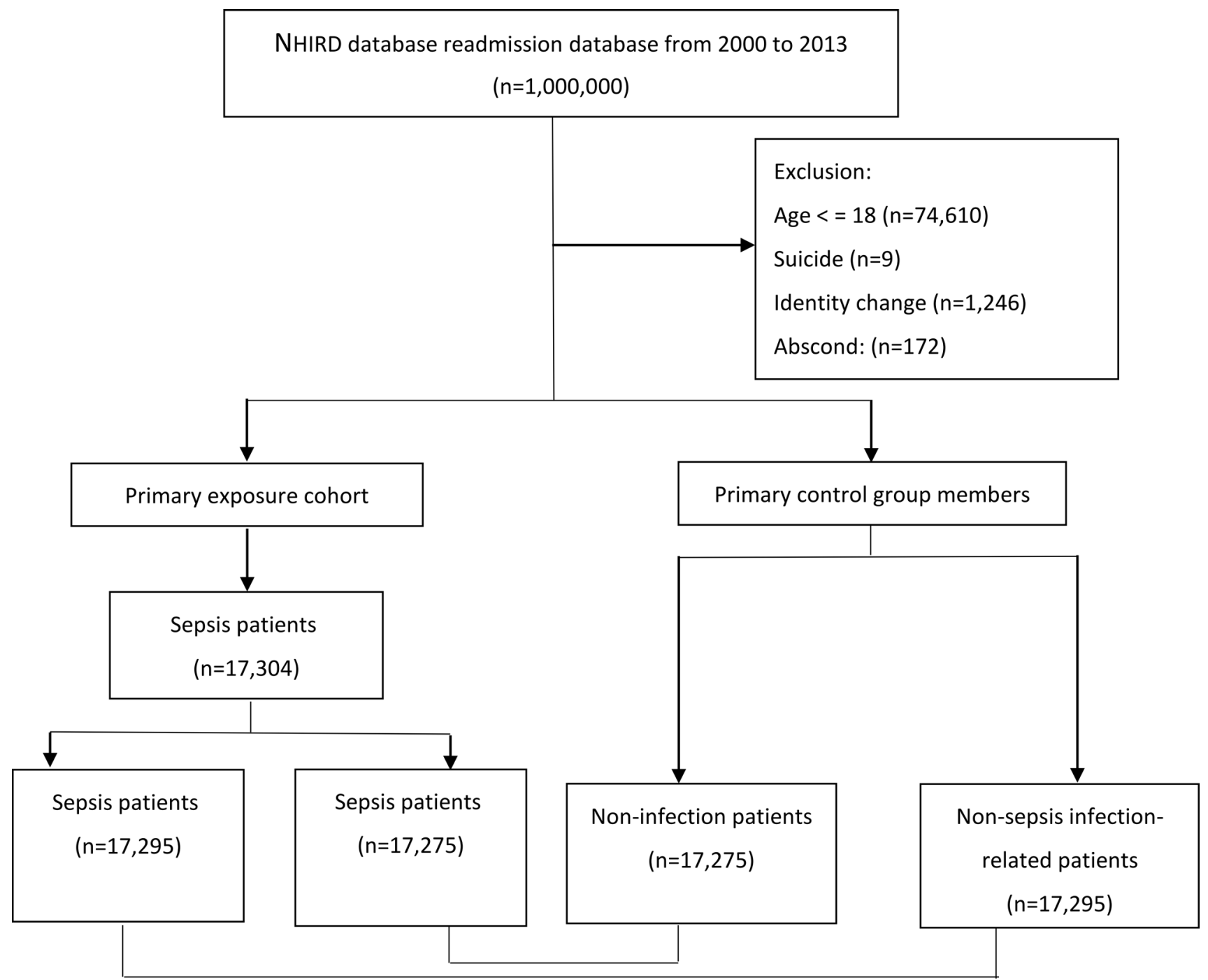

Figure 1 Flow chart of the patient cohort. We first identified sepsis patients using the Martin implementation and then we created three matched cohorts using propensity score method. Two matched cohorts compared sepsis patients with noninfected patients and non-sepsis infected patients. NHIRD, National Health Insurance Research Database.

(427.5), the primary diagnosis code of sudden cardiac death (798) and the presence of the procedure code for cardiopulmonary resuscitation (99.60). The algorithm to identify OHCA has been validated extensively. ${ }^{19}$ We defined patients with an event within 30 days of hospital discharge as early OHCA and patients with an event within 31-365 days as late OHCA. The timeline of the study design and periods of data collection are displayed in online supplemental figure 1.

\section{Data analysis}

We compared baseline characteristics between sepsis survivors who developed OHCA and those who did not. Continuous variables with normal distributions were presented as mean (SD) and compared with Student's t-tests. Categorical variables were described with frequencies and percentages, and then compared using $\chi^{2}$ tests. After comparing the incidence of OHCA between the sepsis and non-sepsis comparison cohorts, we constructed a non-sepsis comparison cohort using the PS matching method mentioned above. We then plotted the daily number and daily risk of OHCA within 1 year of hospital discharge between the sepsis and PS matched non-sepsis cohorts. We used stratified Cox proportional hazard regression analysis to evaluate the risk of early and late OHCA in both the sepsis and non-sepsis cohorts. The proportional hazards assumption was verified by plotting negative $\log$ survival function against $\log$ time. To assess whether patients with certain characteristics were more likely to develop OHCA after sepsis, we conducted subgroup analysis using prespecified clinically important subgroups, including Age ( $>$ or $\leq 75$ years), sex and the presence or absence of comorbid conditions (cancer, cardiovascular disease or diabetes mellitus). All analyses were performed using SAS statistical software V.9.4 (SAS Institute) and followed the Strengthening the Reporting of Observational Studies in Epidemiology reporting guidelines. All tests were two sided, with $\mathrm{p}<0.05$ indicating statistical significance. 


\section{Patient and public involvement}

It was not possible to involve patients or the public in our study. However, NHIRD database was developed with patient and public involvement by the committee at the Ministry of Health and Welfare, Taiwan.

\section{RESULTS}

\section{Study population}

The source cohort in the NHIRD database included 1 million randomly selected patients from the population, who were longitudinally followed from 2000 to 2013 (figure 1). Patients with incomplete clinical data (discharges against medical advice, suicide, transfers out of hospital and otherwise incomplete data) or less than 20 years of age were excluded from the analysis $(n=8028)$. A total of 17304 patients with sepsis were included for analysis, of which 17275 were matched to non-infected patients (comparison cohort 1) and 17295 were matched to non-sepsis infected patients (comparison cohort 2). These groups were matched using PS matching algorithms. The standardised difference of matching covariates between sepsis and non-sepsis patients was less than $10 \%$ (online supplemental figure 2) with a symmetric distribution of PSs after matching (online supplemental figure 3), indicating a successful match.

\section{Patient characteristics}

Online supplemental table 2 compares characteristics of patients who developed OHCA to those who did not develop OHCA within 30 days of hospital discharge, prior to PS matching. A total of 144 sepsis patients developed OHCA within 30 days of hospital discharge. OHCA patients were generally older and the most common comorbidities among both the OHCA and non-OHCA groups were chronic obstructive pulmonary disease, peptic ulcer disease and diabetes. Prior to the sepsis admission, OHCA patients were more likely to have congestive heart failure, atrial fibrillation, neurological disorders and dementia. Sources of infection were similar between groups, but OHCA patients had more lower respiratory tract infections. We performed an additional analysis to compare the characteristics of patients who developed OHCA to those who did not develop OHCA within 365 days of hospital discharge, prior to PS matching (online supplemental table 3). A total of 641 $(3.7 \%)$ sepsis patients developed OHCA within 365 days of hospital discharge.

\section{Risk of OHCA in sepsis patients compared with non-infected patients}

In the first comparison cohort, 17275 sepsis patients were matched to the same number of hospitalised patients without an infection. Within 30 days of hospital discharge, sepsis patients developed 144 OHCAs (Incidence $0.279 / 1000$ person-days) while the control patients without an infection developed 87 OHCAs (incidence 0.17/1000 person-days), with a HR of 1.66 (95\% CI: 1.27 to 2.16). Between 31 and 365 days, sepsis patients developed 640 OHCAs (Incidence 0.104/1000 person-days) while control patients developed 542 OHCAs (incidence $0.087 / 1000$ person-days), with an HR of 1.19 (95\% CI: 1.06 to 1.33 ) (table 1). Patients with sepsis were more likely to experience OHCA than patients in both comparator groups. The daily number of OHCA events among sepsis patients and patients without an infection is shown in figure 2. The HR of OHCA over 365 days comparing sepsis to non-infected patients remained relatively constant over time (figure 3).

\section{Risk of OHCA in sepsis patients compared with non-sepsis patients with infections}

In the second comparison cohort, 17295 sepsis patients were matched to the same number of non-sepsis patients with infections. Within 30 days of hospital discharge, sepsis patients developed 144 OHCAs (incidence 0.279/1000 person-days) while non-sepsis control patients with infections developed 113 OHCAs (incidence 0.219/1000 person-days), with an HR of 1.28 (95\% CI: 1.00 to 1.63 ). Between 31 and 365 days, sepsis patients developed 640 OHCAs (Incidence 0.104/1000 person-days) while control patients developed 566 OHCAs (Incidence

Table 1 Risk of early OHCA ( 0 -30 days postdischarge) and late OHCA (31-365 days postdischarge) after sepsis admission among the propensity score matched cohorts

\begin{tabular}{|c|c|c|}
\hline Time interval for OHCA & $\begin{array}{l}\text { Sepsis versus non-infected hospitalised } \\
\text { patients }\end{array}$ & $\begin{array}{l}\text { Sepsis versus non-sepsis infection- } \\
\text { related patients }\end{array}$ \\
\hline $\begin{array}{l}\text { Early OHCA ( } 0-30 \text { days) incidence } \\
(1 / 1000 \text { person-days) }\end{array}$ & $\begin{array}{l}\text { Sepsis: (144/515 } 726 \text { person-days) } \\
\text { Non-infection: (87/516 } 704 \text { person-days) }\end{array}$ & $\begin{array}{l}\text { Sepsis: (144/516 } 326 \text { person-days) } \\
\text { Non-sepsis: (113/516 } 856 \text { person-days) }\end{array}$ \\
\hline $\begin{array}{l}\text { Early OHCA ( } 0-30 \text { days) } \\
\text { HR }(95 \% \mathrm{Cl})\end{array}$ & $\begin{array}{l}1.66(1.27 \text { to } 2.16)^{\star} \\
\mathrm{P}=0.0002\end{array}$ & $\begin{array}{l}1.28(1.00 \text { to } 1.63) \\
P=0.0500\end{array}$ \\
\hline $\begin{array}{l}\text { Late OHCA ( } 31-365 \text { days) incidence } \\
\text { (1/1000 person-days) }\end{array}$ & $\begin{array}{l}\text { Sepsis: (640/6 } 174507 \text { person days) } \\
\text { Non-sepsis: (542/6 } 202632 \text { person-days) }\end{array}$ & $\begin{array}{l}\text { Sepsis: (640/6 } 181807 \text { person-days) } \\
\text { Non-sepsis:(566/6 } 200964 \text { person-days) }\end{array}$ \\
\hline $\begin{array}{l}\text { Late OHCA ( } 31-365 \text { days) } \\
\mathrm{HR}(95 \% \mathrm{Cl})\end{array}$ & $\begin{array}{l}1.19(1.06 \text { to } 1.33)^{\star} \\
P=0.0035\end{array}$ & $\begin{array}{l}1.13(1.01 \text { to } 1.27)^{\star} \\
P=0.0294\end{array}$ \\
\hline
\end{tabular}

*Means $p<0.05$.

HR, hazard ratio; OHCA, out-of-hospital cardiac arrest. 
A

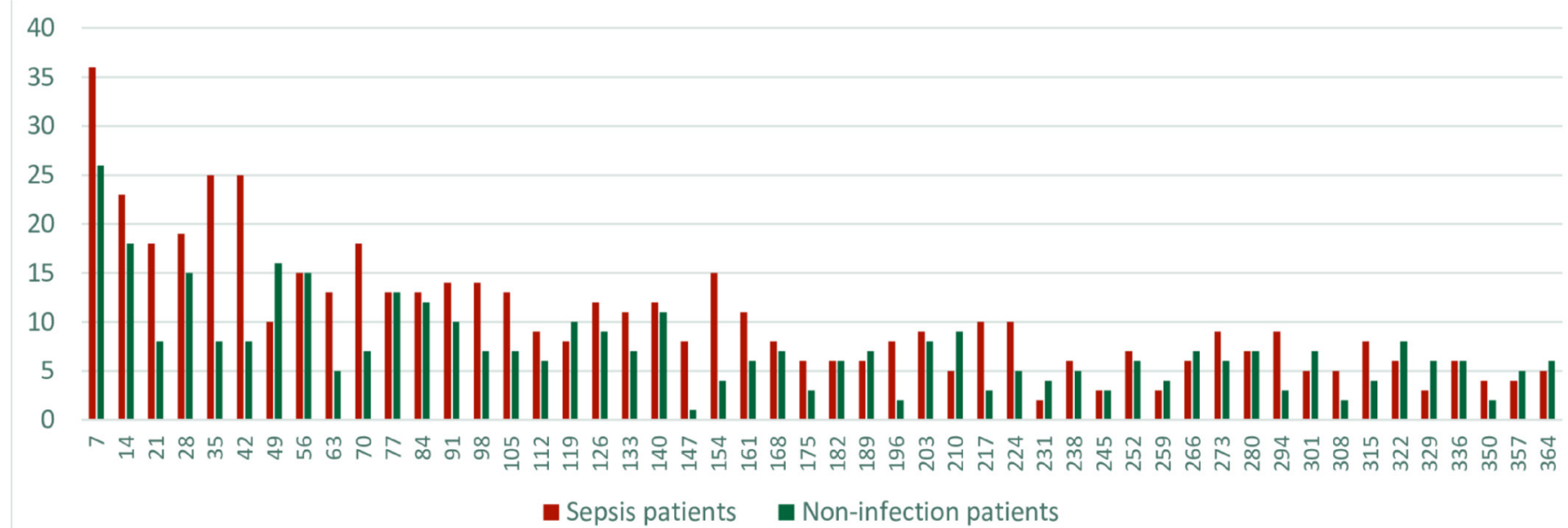

B

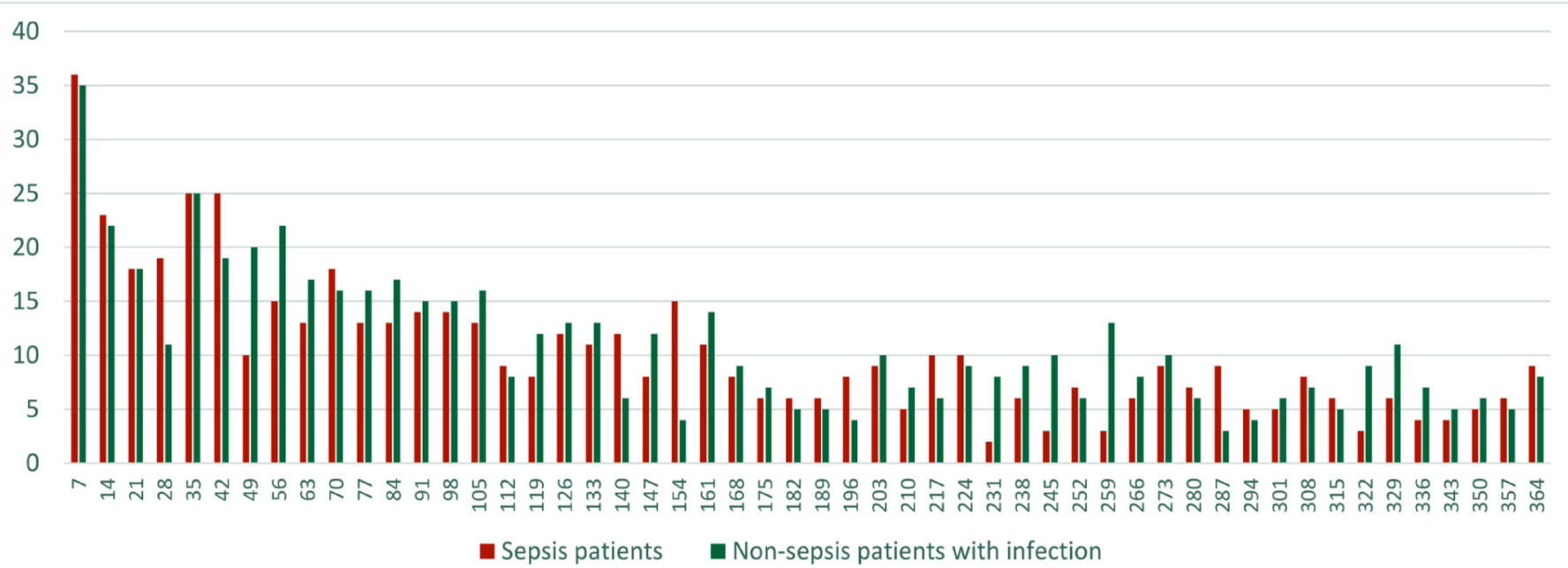

Figure 2 Daily number of out-of-hospital cardiac arrest (OHCA) patients comparing sepsis to non-sepsis patients within 365 days of hospital discharge. Sepsis patients (red) are compared with non-infected patients (green). The number of OHCA events are plotted per week over the span of 1 year. (A) Sepsis patients (red) are compared with non-sepsis patients with infections (green) (B). The number of OHCA events are plotted per week over the span of 1 year.

0.091/1000 person-days), with an HR of 1.13 (95\% CI: 1.01 to 1.27 ) (table 1 ). The daily number of OHCA events among sepsis and non-sepsis patients with infections is shown in figure 2. The HR of OHCA over 365 days comparing sepsis to non-sepsis patients with infections also remained relatively stable over time (figure 3 ). The difference was greatest within 30 days, but data was significant up to 1 year. The HR for sepsis versus non-infected patients was 1.66 (95\% CI: 1.27 to 2.16) at 30 days and 1.19 (95\% CI: 1.06 to 1.33 ) at 1 year. The HR for sepsis vs infected patients without sepsis was 1.28 (95\% CI: 1.00 to $1.63)$ at 30 days and 1.05 (95\% CI: 0.69 to 1.59) at 1 year (table 1).

\section{Risk of risk of OHCA in patients with septic shock}

In a sensitivity analysis, we investigated the risk of OHCA associated with septic shock. Of 17304 sepsis patients, $6244(36.1 \%)$ developed septic shock. The HR for septic shock versus non-infected patients was 1.97 (95\% CI:
1.56 to 2.49 ) at 30 days and 1.05 (95\% CI: 0.69 to 1.59 ) at 1 year. The HR for septic shock versus infected patients without sepsis was 1.80 (95\% CI: 1.11 to 2.94 ) at 30 days and 1.03 (95\% CI: 0.85 to 1.25 ) at 1 year (online supplemental table 4).

\section{Subgroup analysis}

Subgroup analyses were performed within both cohort pairs. In the sepsis versus non-infection arm, patients over the age of 75 (OR 1.70 (95\% CI 1.20 to 2.42)), patients without known cardiovascular disease (OR 3.62 (95\% CI 1.79 to 7.31 ), and patients without diabetes (OR 2.00 (95\% CI 1.43 to 2.81$)$ ) demonstrated a significantly increased risk of OHCA. There was no significant interaction with cancer or sex regarding risk of OHCA from sepsis in this cohort.

In the sepsis versus non-sepsis infection arm, there were fewer differences noted. Only patients with cardiovascular disease (OR 1.35 (95\% CI 1.01 to 1.81)) and non-cancer 

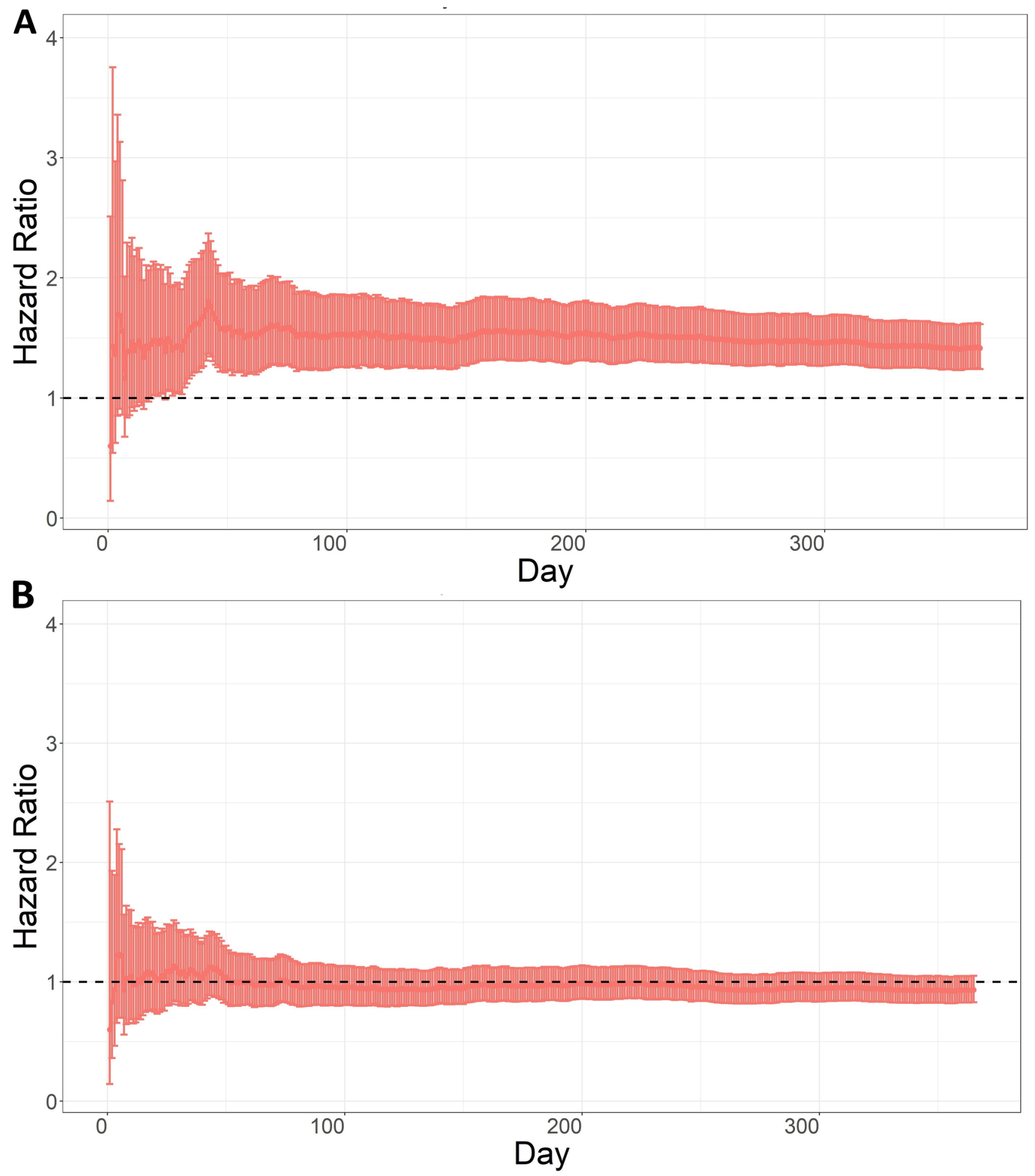

Figure 3 Daily HR 95\% confidence bands of OHCA comparing sepsis to non-infected patients (A) and comparing sepsis to non-sepsis infected patients (B). OHCA, out-of-hospital cardiac arrest.

patients (OR 1.32 (95\% CI 1.01 to 1.81 )) had a statistically significant increased risk of OHCA. In this cohort, there were no significant interactions between age, cancer or diabetes and the risk of OHCA from sepsis.

\section{DISCUSSION}

Increasingly, research is being published exploring the risk of cardiac dysfunction with inflammation and sepsis. ${ }^{20-22}$ While the exact mechanism of sepsis-related cardiac arrest is not well understood, it is thought to be related to the combination of hypovolaemia, metabolic imbalances, hypoxaemia, vasodilation, capillary leak, progression of vascular lesions and myocardial stunning that occurs during a septic event. ${ }^{123}$ It is not unreasonable to conclude that the upregulation of cytokines and prothrombotic factors associated with sepsis in the setting 
of increased myocardial oxygen demand would increase a patient's risk for significant myocardial injury. ${ }^{20} 24$

To our knowledge, our study is the first large, nationally representative analysis to show an increased rate of OHCA among patients previously hospitalised with sepsis. The risk for OHCA persisted for at least 1year after discharge with a peak within the first 100 days. The results of our research are consistent with prior studies that have demonstrated associations between infection and adverse cardiovascular events, such as stroke and myocardial infarction..$^{25}$ For example, it is well established that patients with pneumonia experience acute coronary syndromes (ACS) proportional in severity to their concomitant infection, and even 10 years after discharge, they suffer from higher rates of ACS than their comorbidity-matched peers. ${ }^{26}{ }^{27}$ Similar (although less protracted) trends are observed among patients hospitalised with urinary tract infections (UTIs) ${ }^{28}$ With the insight that pulmonary and urinary infections are associated with higher rates of ACS, it is not surprising that the sepsis patients with pneumonia and UTIs in our study represented the two most common types of infection in the OHCA population. While further investigations are needed to fully understand the pathophysiology of sepsis-induced cardiac dysfunction (whether specific to pneumonia or to the inflammatory response at large), we hope that with more studies like ours, it will be difficult to ignore the need to further delve into the relationship between sepsis and adverse cardiac events.

A major strength of our study was that we were able to use a large, nationally representative longitudinal sample population to identify cases and extract information about relatively rare events with sufficiently powered and matched controls. However, a few important limitations in our study must be noted. First, due to the observational nature of the study, causality could not be established. Second, the use of an administrative database might have limited our possibility to capture important characteristics contributing to each patient's unique presentation such as the magnitude of sepsis severity (eg, classification of patients according to the Sequential Organ Failure Assessment score) and whether the patient presented to the ED with sepsis or developed an infection after being hospitalised for several days. As with other claims databases, data on some lifestyle risk factors such as smoking were not available. Whenever possible, we employed proxy measures (eg, chronic obstructive pulmonary disease) to account for these lifestyle factors. In spite of this, there may be further validity concerns in regards to the accuracy of diagnostic codes and unmeasured confounders. ${ }^{29}$ To address these concerns, the Taiwanese Ministry of Health and Welfare has been working to centralise management and analysis of the NHIRD database with regular updates to validate codes and identify unmeasured confounders to more directly enhance the reliability of NHIRD studies. ${ }^{29}$

While this study strongly suggests a relationship between sepsis and the subsequent development of OHCA, the associations identified are limited by the reliance on cohort data. To that end, more studies are needed to assess the impact of sepsis on OHCA populations. For example, direct anatomopathological classification of myocardial injuries could be investigated to establish whether sepsis survivors showed signs of excess inflammation, remodelling or atheroembolic events. ${ }^{30}$ Alternatively, no randomised controlled trials have investigated predischarge interventions aimed at reducing cardiovascular risk among patients hospitalised with sepsis. Perhaps interventions could be designed that randomise sepsis survivors to receive 1 year of primary and secondary preventive pharmacotherapies to determine the extent to which sepsis-related OHCA responds to typical cardiovascular risk-reducing strategies. Previous studies disclosed the association between sepsis and cardiovascular events, including stroke and myocardial infarction. ${ }^{31-33}$ Therefore, the cardiovascular outcomes developed after sepsis discharge can be summarised as 'major adverse cardiac events (MACE)'. Secondary pharmacological prevention in patients with MI has been shown effective. Therefore, it is reasonable to see if a similar preventive strategy may reduce MACE in sepsis survivors. Given OHCA are overwhelmingly fatal and the potential for preventive interventions is within reach, further studies are urgently needed to more fully investigate these important relationships.

\section{CONCLUSION}

Our population-based database study demonstrated an increased risk for OHCA for sepsis patients, which extended up to 1 year following hospital discharge. Future clinical studies are needed to better understand the aetiology of cardiovascular dysfunction after a septic event to allow for adoption of effective primary prevention plans.

\section{Author affiliations}

${ }^{1}$ Department of Epidemiology, Harvard University T H Chan School of Public Health, Boston, Massachusetts, USA

${ }^{2}$ Department of Medicine, Warren Alpert Medical School of Brown University, Providence, Rhode Island, USA

${ }^{3}$ Department of Emergency Medicine, Università degli Studi di Milano-Bicocca, Milano, Italy

${ }^{4}$ Department of Emergency Medicine, National Taiwan University Hospital, Taipei, Taiwan

${ }^{5}$ Department of Internal Medicine, National Taiwan University Hospital, Taipei, Taiwan

${ }^{6}$ The Centre for Intelligent Healthcare, National Taiwan University Hospital, Taipei, Taiwan

${ }^{7}$ Byers Center for Biodesign, School of Medicine, Stanford University, Palo Alto, CA, USA

\section{Collaborators None.}

Contributors All authors participated substantially in the manuscript and met the ICMJE authorship criteria. All authors take responsibility for all aspects of the reliability and freedom from bias of the data presented and their discussed interpretation. W-TH: conceptualisation, investigation, writing-reviewing and editing, supervision. CFS: writing-reviewing and editing. BT: writing-reviewing and editing. AP: writing-reviewing and editing. LP: writing-reviewing and editing. T-CH: formal analysis, project administration. W-HS: writing-reviewing and editing. C-CL: conceptualisation, methodology, data curation, writing-original draft, writingreviewing and editing, supervision, funding acquisition. 
Funding This study was funded by National Taiwan University Hospital Grant NTUH.110-s4891. No funding bodies had any role in study design, data collection and analysis, decision to publish, or preparation of the manuscript.

Competing interests None declared.

Patient consent for publication Not required.

Ethics approval This study was approved by the institutional review board of National Taiwan University Hospital (201402032RINA). Informed consent was waived given that this is an electronic database study using anonymous subjects.

Provenance and peer review Not commissioned; externally peer reviewed.

Data availability statement № data are available. Raw data were generated at National Taiwan University Health Data Research Center. No additional data are available.

Supplemental material This content has been supplied by the author(s). It has not been vetted by BMJ Publishing Group Limited (BMJ) and may not have been peer-reviewed. Any opinions or recommendations discussed are solely those of the author(s) and are not endorsed by BMJ. BMJ disclaims all liability and responsibility arising from any reliance placed on the content. Where the content includes any translated material, BMJ does not warrant the accuracy and reliability of the translations (including but not limited to local regulations, clinical guidelines, terminology, drug names and drug dosages), and is not responsible for any error and/or omissions arising from translation and adaptation or otherwise.

Open access This is an open access article distributed in accordance with the Creative Commons Attribution Non Commercial (CC BY-NC 4.0) license, which permits others to distribute, remix, adapt, build upon this work non-commercially, and license their derivative works on different terms, provided the original work is properly cited, appropriate credit is given, any changes made indicated, and the use is non-commercial. See: http://creativecommons.org/licenses/by-nc/4.0/.

ORCID iD

Wan-Ting Hsu http://orcid.org/0000-0002-6677-0951

\section{REFERENCES}

1 Desai R, Parekh T, Patel U, et al. Regional trends in in-hospital cardiac arrest following sepsis-related admissions and subsequent mortality. Resuscitation 2019;143:35-41.

2 Carr GE, Yuen TC, McConville JF, et al. Early cardiac arrest in patients hospitalized with pneumonia: a report from the American heart association's get with the Guidelines-Resuscitation program. Chest 2012;141:1528-36.

3 Chang C-J, Liou T-H, Tsai W-T, et al. Clinical and hematological predictors for return of spontaneous circulation in patients with outof-hospital cardiac arrest. J Acute Med 2020;10:51.

4 Andersen LW, Holmberg MJ, Berg KM, et al. In-Hospital cardiac arrest: a review. JAMA 2019;321:1200-10.

5 Chan PS, McNally B, Tang F, et al. Recent trends in survival from out-of-hospital cardiac arrest in the United States. Circulation 2014;130:1876-82.

6 Morgan RW, Fitzgerald JC, Weiss SL, et al. Sepsis-associated inhospital cardiac arrest: epidemiology, pathophysiology, and potential therapies. J Crit Care 2017;40:128-35.

7 Ballew KA, Philbrick JT, Caven DE, et al. Predictors of survival following in-hospital cardiopulmonary resuscitation. A moving target. Arch Intern Med 1994;154:2426-32.

8 Taffet GE, Teasdale TA, Luchi RJ. In-hospital cardiopulmonary resuscitation. JAMA 1988;260:2069-72.

9 Angus DC, Linde-Zwirble WT, Lidicker J, et al. Epidemiology of severe sepsis in the United States: analysis of incidence, outcome, and associated costs of care. Crit Care Med 2001;29:1303-10.
10 Mayr FB, Talisa VB, Balakumar V, et al. Proportion and cost of unplanned 30-day readmissions after sepsis compared with other medical conditions. JAMA 2017;317:530-1.

11 Martin GS, Mannino DM, Eaton S, et al. The epidemiology of sepsis in the United States from 1979 through 2000. N Engl J Med 2003;348:1546-54.

12 Singer M, Deutschman CS, Seymour CW, et al. The third International consensus definitions for sepsis and septic shock (Sepsis-3). JAMA 2016;315:801-10.

13 Lee C-C, Lee M-TG, Lee W-C, et al. Preadmission use of calcium channel blocking agents is associated with improved outcomes in patients with sepsis: a population-based propensity score-matched cohort study. Crit Care Med 2017;45:1500-8.

14 Hsu W-T, Galm BP, Schrank G, et al. Effect of renin-angiotensinaldosterone system inhibitors on short-term mortality after sepsis: a population-based cohort study. Hypertension 2020;75:483-91.

15 Yu C-W, Chang S-S, Lai C-C, et al. Epidemiology of emergency department sepsis: a national cohort study between 2001 and 2012 Shock 2019;51:619-24.

16 Yo C-H, Lai C-C, Hsu T-C, et al. National trends of organ dysfunctions in sepsis:an 11-year longitudinal population-based cohort study. J Acute Med 2019;9:178.

17 Charlson ME, Pompei P, Ales KL, et al. A new method of classifying prognostic comorbidity in longitudinal studies: development and validation. J Chronic Dis 1987;40:373-83.

18 Halanych JH, Safford MM, Keys WC, et al. Burden of comorbid medical conditions and quality of diabetes care. Diabetes Care 2007;30:2999-3004.

19 Shelton SK, Chukwulebe SB, Gaieski DF, et al. Validation of an ICD code for accurately identifying emergency department patients who suffer an out-of-hospital cardiac arrest. Resuscitation 2018;125:8-11.

20 Fenton KE, Parker MM. Cardiac function and dysfunction in sepsis. Clin Chest Med 2016;37:289-98.

21 Merx MW, Weber C. Sepsis and the heart. Circulation 2007;116:793-802.

22 Bosmann M, Ward PA. The inflammatory response in sepsis. Trends Immunol 2013;34:129-36.

23 Ramirez J, Aliberti S, Mirsaeidi M, et al. Acute myocardial infarction in hospitalized patients with community-acquired pneumonia. Clin Infect Dis 2008;47:182-7.

24 Mankowski RT, Yende S, Angus DC. Long-Term impact of sepsis on cardiovascular health. Intensive Care Med 2019;45:78-81.

25 Corrales-Medina VF, Alvarez KN, Weissfeld LA, et al. Association between hospitalization for pneumonia and subsequent risk of cardiovascular disease. JAMA 2015;313:264-74.

26 Jafarzadeh SR, Thomas BS, Warren DK, et al. Longitudinal study of the effects of bacteremia and sepsis on 5-year risk of cardiovascular events. Rev Infect Dis 2016;63:495-500.

27 Smeeth L, Thomas SL, Hall AJ, et al. Risk of myocardial infarction and stroke after acute infection or vaccination. $N$ Engl J Med Overseas Ed 2004;351:2611-8.

28 Fukuda T, Ohashi-Fukuda N, Kondo Y, et al. Epidemiology, risk factors, and outcomes of out-of-hospital cardiac arrest caused by stroke. Medicine 2016;95:e3107.

29 Hsieh C-Y, Su C-C, Shao S-C, et al. Taiwan's National health insurance research database: past and future. Clin Epidemiol 2019;11:349-58.

30 Musher DM, Abers MS, Corrales-Medina VF. Acute infection and myocardial infarction. N Engl J Med Overseas Ed 2019;380:171-6.

31 Lai C-C, Lee M-tseG, Lee W-C, et al. Susceptible period for cardiovascular complications in patients recovering from sepsis. Can Med Assoc J 2018;190:E1062-9.

32 Hsieh Y-C, Tsou P-Y, Wang Y-H, et al. Risk factors for myocardial infarction and stroke among sepsis survivors: a competing risks analysis. J Intensive Care Med 2020;35:34-41.

$33 \mathrm{M}-\mathrm{H}$ W, Tsou P-Y, Wang Y-H. Impact of post-sepsis cardiovascular complications on mortality in sepsis survivors: a population-based study. Critical Care 2019;23:1-11. 